\title{
Assessing the burden of paediatric influenza in Europe: the European Paediatric Influenza Analysis (EPIA) project
}

\author{
W. John Paget $\cdot$ Catherine Balderston • \\ Inmaculada Casas • Gé Donker • Laurel Edelman • \\ Douglas Fleming • Amparo Larrauri • Adam Meijer • \\ Simona Puzelli • Caterina Rizzo • Lone Simonsen • \\ and all EPIA collaborators
}

Received: 30 October 2009 /Accepted: 4 February 2010 / Published online: 13 March 2010

(C) The Author(s) 2010. This article is published with open access at Springerlink.com

\begin{abstract}
The European Paediatric Influenza Analysis (EPIA) project is a multi-country project that was created to collect, analyse and present data regarding the paediatric influenza burden in European countries, with the purpose of providing the necessary information to make evidencebased decisions regarding influenza immunisation recommendations for children. The initial approach taken is based
\end{abstract}

EPIA collaborators (SC indicates a Steering Committee Member): Catherine Balderston, Antonino Bella, Pilar Pérez-Breña, Inmaculada Casas, Teresa Lopez Cuadrado, Salvador de Mateo, Isabella Donatelli, Gé Donker, Laurel Edelman, Alex Elliot, Douglas Fleming, Steffen Glisman, Terho Heikkinen (SC), Amparo Larrauri, Raul Ortiz de Lejarazu Leonardo, Anne Mazick, Jim McMenamin, Adam Meijer, Kare Molbak, Lars Nielsen, John Paget, Rajinder Pnaiser, Fabrizio Pregliasco, Simona Puzelli, Arlene Reynolds, Caterina Rizzo (SC), Lone Simonsen, Derek Smith (SC), Tomás Pumarola Suñé, Marianne van der Sande, Tomás Vega Alonso, Timo Vesikari, John Watson, Catherine Weil-Olivier (SC), Maria Zambon (SC), Thedi Ziegler.

W. J. Paget $\cdot$ G. Donker

Netherlands Institute for Health Services Research (NIVEL),

Utrecht, The Netherlands

C. Balderston $(\bowtie) \cdot$ L. Edelman $\cdot$ L. Simonsen

SDI,

Plymouth Meeting, PA, USA

e-mail: kbalderston@sdihealth.com

I. Casas

National Center for Microbiology, Instituto de Salud Carlos III,

Madrid, Spain

D. Fleming

RCGP Research and Surveillance Centre,

Birmingham, UK

\section{A. Larrauri}

National Center for Epidemiology, Instituto de Salud Carlos III,

Madrid, Spain on existing weekly virological and age-specific influenzalike illness (ILI) data from surveillance networks across Europe. We use a multiple regression model guided by longitudinal weekly patterns of influenza virus to attribute the weekly ILI consultation incidence pattern to each influenza (sub)type, while controlling for the effect of respiratory syncytial virus (RSV) epidemics. Modelling the
A. Meijer

Centre for Infectious Disease Control,

National Institute of Public Health and the Environment,

Bilthoven, The Netherlands

S. Puzelli

Department of Infectious, Parasitic and Immune-mediated Diseases, Istituto Superiore di Sanita,

Rome, Italy

C. Rizzo

National Center for Epidemiology, Surveillance and Health Promotion, Istituto Superore di Sanità,

Rome, Italy

L. Simonsen

School of Public Health and Health Services,

George Washington University,

Washington, DC, USA 
ILI consultation incidence during 2002/2003-2008 revealed that influenza infections that presented for medical attention as ILI affected between $0.3 \%$ and $9.8 \%$ of children aged 0 4 and 5-14 years in England, Italy, The Netherlands and Spain in an average season. With the exception of Spain, these rates were always higher in children aged $0-4$ years. Across the six seasons analysed (five seasons were analysed from the Italian data), the model attributed 47 $83 \%$ of the ILI burden in primary care to influenza virus infection in the various countries, with the $\mathrm{A}(\mathrm{H} 3 \mathrm{~N} 2)$ virus playing the most important role, followed by influenza viruses $\mathrm{B}$ and $\mathrm{A}(\mathrm{H} 1 \mathrm{~N} 1)$. National season averages from the four countries studied indicated that between $0.4 \%$ and $18 \%$ of children consulted a physician for ILI, with the percentage depending on the country and health care system. Influenza virus infections explained the majority of paediatric ILI consultations in all countries. The next step will be to apply the EPIA modelling approach to severe outcomes indicators (i.e. hospitalisations and mortality data) to generate a complete range of mild and severe influenza burden estimates needed for decision making concerning paediatric influenza vaccination.

Keywords Influenza $\cdot$ Children · Paediatric $\cdot$ Burden of disease $\cdot$ Modelling $\cdot$ Europe

\section{Introduction}

Influenza A and B viruses cause winter-seasonal epidemics of respiratory illness in the northern hemisphere that have been reported to affect $5-15 \%$ of the population each winter [40], with reports of paediatric attack rates between $20 \%$ and $35 \%$ depending on season and methodology. Symptoms of the disease vary from mild (e.g. requiring little or no treatment) to severe (e.g. requiring hospitalisation). Though death is more common in the elderly and populations that have a higher risk of developing complications following an influenza virus infection (e.g. persons with chronic heart and lung diseases, diabetes, immunodeficiency, pregnancy etc.), severe disease and some deaths occur each season in healthy young and middle-aged adults and children.

Recommendations to vaccinate high-risk populations and persons over 65 years have been implemented in Europe since the $1980 \mathrm{~s} / 1990 \mathrm{~s}(1999 / 2000$ season in the UK) $[7,24,33]$. With minor exceptions, vaccination recommendations are broadly similar across Europe, with most countries recommending the vaccination of persons over 65 years and high-risk groups. In Hungary, Germany and The Netherlands, the age threshold is 60 years; in Finland (since 2007), vaccination of children aged 6-35 months is recommended; in Austria, vaccination is recommen- ded for persons of all ages [27]. All countries in Europe aim to meet the WHO Assembly goal of vaccinating $75 \%$ of high-risk groups by 2010. Results of a regional (European Union and European Economic Area) survey published in 2008 indicated that influenza vaccination coverage rates in the elderly (i.e. $\geq 65$ years) varied substantially from less than $10 \%$ in some countries to over $70 \%$ in others [27].

Several countries, including Argentina, Canada, Finland, Mexico, Singapore and the USA, have recently extended their vaccination recommendations to include younger age groups [41]. These decisions were based, in part, on evidence demonstrating that influenza has substantial adverse impacts among school-age children and their contacts (e.g. significant school absenteeism, antibiotic use, medical care visits and parental work loss) compared to uninfected or asymptomatic children [5, 15, 17, 28]. Additionally, studies have suggested that vaccination of children has the potential to reduce influenza among their household contacts and within the community, thereby reducing the risk of influenza even in high-risk groups (herd immunity) $[18,32,39]$. The US recommendations have recently evolved from 6 to 23 months in 2004 to 6-59 months in 2006 to now include all children up to 18 years of age. The increasing focus on paediatric age groups was in large part fuelled by the heightened awareness of paediatric influenza mortality starting with the severe 2003-2004 Fujian A (H3N2) season $[2,3]$.

In 2007, a scientific panel on vaccines and immunisation, in conjunction with the European Centre for Disease Prevention and Control (ECDC), published a technical report on routine influenza vaccination in children [36]. The report emphasised that the first step towards any decision regarding routine immunisation in children is to determine specific national profiles of the disease burden. Estimates of the burden of influenza in children using sentinel practice data from England and Wales suggested that in a typical winter epidemic between 1987/1988 and 1996/1997, 1.2\% of children aged $0-4$ and $1.1 \%$ of children aged 5-14 years sought medical attention and were diagnosed with ILI; in addition, many more children were diagnosed with other respiratory syndromes such as acute bronchitis and otitis media [14]. The same study using similar data from The Netherlands estimated that $3.0 \%$ of children $0-4$ years and $1.9 \%$ of children $5-14$ years had medically attended ILI in a season. Paradoxically, the Dutch general practitioners, who reported a higher seasonal ILI percentage, worked with a stricter case definition than in the UK.

Because the ECDC report concluded that paediatric disease burden estimates were scarce in most European countries and that this would hamper the ability of countries to make policy decisions about the influenza vaccine, the European Paediatric Influenza Analysis (EPIA) project was 
created in 2008 to collect, analyse and present paediatric influenza burden data in countries across Europe. The approach taken by EPIA was to base the modelling on the robust virological and epidemiological surveillance networks in European countries to generate country-specific disease burden estimates for mild illness (ILI), severe illness (e.g. pneumonia hospitalisations) and mortality. Here, we present the EPIA project, its methods, planned studies and results so far.

\section{The EPIA project}

\section{Background}

Table 1 outlines the different types of data that have been (or will be in the future) collected by EPIA. The project was formed around the European Influenza Surveillance Scheme (EISS) [10], and the first modelling work has centred on the ILI data and virological data collected by this network from 1996 up to 2008. Both the ILI and virological data were collected weekly for the winter periods (i.e. seasons) from week 40 (October) to week 20 (May) of the following year. Data have been collected according to a case definition (in most countries), and the sentinel physicians took nose/throat swabs from a selection of their patients and sent the specimens to their National Influenza Centre to validate the clinical reports made by the sentinel physicians and to determine virus characteristics (i.e. (sub)type) which might impact the severity of disease and extent of virus circulation (e.g. Arkema et al. [1]). While multiple ILI consultations in the context of a single episode are not reported by physicians, multiple episodes for the same patient are reported on occasion but would likely represent a small effect on the incidence estimates reported in the current study.

The virological data were obtained from weekly sentinel and non-sentinel reports from National Influenza Centres, with the sentinel data coming from the sentinel surveillance systems and the non-sentinel specimens coming from other sources (e.g. hospital and peripheral diagnostic laboratories). As most sentinel specimens have been tested for influenza and RSVs, with the exception of Spain, the National Influenza Centres provide weekly data on both viruses. Within three of the four country datasets (England, The Netherlands and Spain), the majority of the RSV data were non-sentinel in nature (in The Netherlands and Spain, these data were obtained from separate surveillance systems: the Dutch Working Group on Clinical Virology in The Netherlands and the Microbiological Information System in Spain), but valid inclusion was assumed based on published reports of these isolates being derived from mostly children $[14,16,38]$. Laboratories within the EISS system regularly participated in quality control assessments reported elsewhere $[25,26]$. RSV is a virus that often represents a confounding variable in influenza research of this type as its resulting infection can produce similar symptoms and the virus often circulates in a seasonal pattern along with influenza viruses [11]. Whilst the ILI data are age specific $(0-4,5-14,15-64$ and $65+)$, the virological data were only available for all ages; for the purpose of EPIA modelling, we have assumed that all-age epidemic patterns for influenza virus and RSV detections are representative of epidemic patterns (intensity and duration) in the paediatric age groups, a generally accepted method within this area of research (e.g. [13, 14, 19, 30]).

To estimate the contribution of moderate and severe outcomes due to influenza virus and RSV infections, EPIA is also collecting weekly or monthly hospital (and, in the case of Denmark, emergency department) discharge data from each country. To this end, EPIA has developed a list of influenza-relevant ICD-9 and ICD-10 code ranges and submitted requests for extraction of data, stratified by up to 24 age groups. These ICD-coded definitions are also being used to obtain mortality data from each country. The current study focuses entirely on the modelled burden of physician-intended ILI in children (in the outpatient setting). Analyses of inpatient and mortality data will be published separately.

\section{Participating countries}

All European countries are invited to participate in the EPIA project provided they have several years of virological data (weekly counts of subtyped influenza viruses and RSVs) and data from at least one outcome of interest; for example, ILI or inpatient data. Currently, the following seven countries participate in the EPIA project: Denmark, England, Finland, Italy, The Netherlands, Scotland and Spain. In this paper, we report results from a subset of EPIA member countries for which the ILI data and virology data had been obtained, cleaned, analysed and discussed with the country collaborators.

\section{Management and underlying sponsorship}

The private-public EPIA collaborative project is funded by MedImmune, the wholly owned biologics business for AstraZeneca PLC. The project is being implemented by SDI, a US market insight and analytics firm that consolidates electronic healthcare data (e.g. RSV surveillance in the USA [4]) for clients in the private and public sectors. The partner, NIVEL, is located in The Netherlands and is a not-for-profit research foundation. NIVEL is responsible for coordinating EPIA activities in Europe based on its experience with the coordination of the EISS from November 1999 to September 2008 [10]. 
Table 1 Data types collected by EPIA for disease and pharmacoeconomic burden estimation

Weekly outpatient visits from sentinel physicians (ILI data)

Weekly virological data from sentinel and non-sentinel sources (influenza and RSV data)

Weekly or monthly hospitalisation data: pneumonia, sepsis and more; outcomes may include numbers of visits, hospital days and the cost of the event

Weekly or monthly emergency department data

Weekly or monthly antibiotic utilisation data

Weekly or monthly mortality data: influenza and pneumonia, sepsis and more

Age strata <3, 3-5, 6-12 months; 1, 2, 3, 4, 5-9, 10-14, 15-19, 20-24, 25-29, 30-34, 35-39, 40-44, 45-49, 50-54, 55-59, 60-64, 65-69, 70$74,75-79,80-84, \geq 85$ years old

EPIA ensures that the research, analysis and results are clearly independent of its underlying sponsor through the following measures:

1. SDI, NIVEL and MedImmune have signed a Memorandum of Understanding which ensures that MedImmune is not involved in the study design, analysis, publication (aside from pre-publication review of the final manuscript) or management of the EPIA project (http://www.nivel.eu/epia/).

2. An independent Steering Committee (see below) has been established to supervise the EPIA project and ensure, that the Memorandum of Understanding is followed.

3. Documents detailing the guiding principles enumerated in Box 1 were agreed upon by EPIA members. These principles clearly define the operating procedures with respect to data analyses, authorship, data ownership and conflict resolution.

The EPIA Steering Committee is comprised of five persons from various European Union Member States representing the following fields: influenza researchers, paediatricians, epidemiologists and virologists. In addition, a liaison from ECDC participates in Steering Committee and member meetings.

Box 1 Guiding principles of the European Paediatric Influenza Analysis project

\section{Guiding principles}

- Data ownership/sharing is decided by members or according to rules of data sources (e.g. private sector data)

- EPIA is open to all countries in Europe

- Models are shared with national collaborators

- Multi-country and national publications are supported

- EPIA is a publication-driven project
- EPIA is a research project, not a surveillance project

- A Memorandum of Understanding between the sponsor and the project managers ensures the sponsor does not influence data analysis or interpretation

- The sponsor does not have access to the EPIA data

- The project is guided by an independent Steering Committee

\section{Methods}

Description of data and data sources utilised in the current study

The underlying surveillance effort for each country is described here; the ILI case definitions are tabulated below (Table 2).

England The Royal College of General Practitioners Weekly Returns Service (WRS) includes approximately 500 general practitioners (GPs) covering 1.8\% of the entire population and is representative of age, sex, socioeconomic status and ethnicity [12]. During winter, network GPs are asked to collect nasal/nasopharyngeal specimens from a sample of ILI patients for virological testing. Over each of the last five winters approximately 1,000 samples have been submitted for virological determination. The WRS collects incident case data from age- and gender-specific defined populations for all diagnoses including ILI. Participating doctors are given guidance on diagnosing specific diseases but are asked to record the diagnosis using the diagnostic term most appropriate to the clinical presentation.

Italy The Italian epidemiological surveillance network consists of 762 sentinel GPs and 213 paediatricians reporting ILI cases on a weekly basis during the winter 
Table 2 Case definitions of influenza-like-illness employed by EPIA countries included in this paper

\begin{tabular}{ll}
\hline Surveillance network & Case definition \\
\hline England & No absolute case definition but physicians are advised to include illness with a sudden onset, fever or \\
& feverishness, one or more respiratory and one or more systemic symptom (e.g. headache, muscle pains) \\
& Sudden onset of symptoms, with temperature $>38^{\circ} \mathrm{C}$, plus at least one of the following systemic symptoms: \\
headache, malaise, chills, sweats, fatigue; plus at least one respiratory symptom (cough, sore throat, or \\
nasal obstruction) \\
Note: In very young children, physicians are advised to consider that (1) they are not able to describe \\
symptoms and often have nonspecific signs such as: irritability, anorexia, crying; (2) vomiting and \\
diarrhoea are common symptoms in neonates, while fever is uncommon; (3) conjunctivitis is often \\
associated with influenza in pre-school children; and (4) in children between 1 and 5 years of age, \\
the most important influenza symptoms are fever with laryngitis and/or bronchitis \\
An acute onset (at most a prodromal stage of 3 to 4 days), accompanied by a rise in rectal temperature of \\
$>38^{\circ} \mathrm{C}$, and at least 1 of the following symptoms: cough, coryza, sore throat, frontal headache, retrosternal \\
pain, myalgia \\
At least 4 of the following characteristics in the context of an influenza epidemic (6 criteria are needed in the \\
absence of an influenza epidemic): acute onset (within 12 hours), cough, fever, chills, prostration and \\
weakness, myalgia or general pain, rhinitis, pharyngitis, contact with a case
\end{tabular}

season, representing $2 \%$ of the population, representative for age, geographic distribution and urbanisation level. Each participating doctor is given instructions on which day to take the swabs; they collect samples from the first ILI cases observed during the pre-specified days. The average number of samples collected since 2000 was 2,500 per season.

The Netherlands The Dutch network consists of approximately 65 GPs (across 45 practices) covering about 1\% of the total population, which is representative of age, sex, geographic distribution and urbanisation level. During winter, sentinel GPs are asked to collect nasal/nasopharyngeal specimens from randomly selected patients with ILI for virological testing. These physicians are asked to collect specimens from at least two patients per week preferentially including one younger than 10 years of age, irrespective of symptom severity. Network-wide averages of approximately 20 specimens are collected every week during the season.

Spain The Spanish Influenza Sentinel Surveillance System consists of about 497 GPs and 171 paediatricians covering approximately $2 \%$ of the national population and is representative of age, sex and urbanisation level. During winter, sentinel GPs and paediatricians collected nasal/nasopharyngeal specimens from a subset of ILI patients, yielding about 35 samples per week for virological testing [23].

\section{Modelling}

A direct assessment of the influenza disease burden is not possible because influenza virus infection is rarely confirmed by laboratory testing and because a triggering influenza virus infection may have resolved by the time a patient presents with secondary complications (e.g. bacterial pneumonia). Instead, inferential statistical methods (e.g. regression modelling) and outcome data (e.g. physician consultations diagnosed as ILI) are commonly used to estimate the seasonal disease burden specific to influenza virus infection (i.e. to understand how ILI rates vary with any one of the independent viral variables).

Modelling the paediatric burden of influenza is further complicated by the concurrent impact of other important respiratory pathogens, especially $\mathrm{RSV}$, which often cocirculates with influenza viruses during winter months. Importantly, the model is capable of estimating the contribution of influenza virus infection to physicians' ILI consultation incidence while controlling for the effects of RSV on the dependent variable (i.e. weekly ILI rate). The model was created to estimate the seasonal influenza burden in paediatric age groups; in this paper, data for children $0-4$ and 5-14 years of age are presented. However, the model may also be applied to adult age groups and could also be used to generate burden estimates for the ongoing $2009 \mathrm{~A}(\mathrm{H} 1 \mathrm{~N} 1)$ pandemic in near real time for medically attended illness (e.g. ILI, pneumonia and/or influenza).

Four participating countries with readily available ILI and linked virologic data needed for the modelling were used to develop the multiple regression model employed in the current study. All available seasons where virological data were stable (i.e. seasons with simultaneous surveillance of both influenza virus and RSV) were analysed. Additionally, two sensitivity analyses were conducted. The first analysis was undertaken to investigate the consequence of overlapping RSV and influenza epidemics by restricting the Dutch data to three seasons (2002-2005) where 
epidemics of influenza and RSV did not occur simultaneously. The second sensitivity analysis was conducted to assess the effects of incomplete RSV virology data on model estimates of ILI attributed to RSV by restricting the Italian data to THE two seasons (2005-2007) which contained the majority of RSV-positive test reports. In the next phase of the project, EPIA collaborators will apply the model to data from moderate and severe outcomes (e.g. hospitalisation and mortality), while modifying the regression equation to account for secular and seasonal patterns in the data. The influenza burden estimates derived from the model will then be applied in the pharmacoeconomic analysis, enabling elucidation of the financial consequences of the disease.

A multiple linear regression model approach was generated in both SAS (the GENMOD procedure) and Stata (the GLM command) [34, 35] formats and shared with EPIA member countries along with the offer of statistical and publishing support. The goal is for the individual countries to carry out subsequent national analyses (using their country-specific data) and publish the results. The modelling approach described below was chosen to capitalise on the existing viral surveillance data and to allow for investigation of individual virus (sub)types. Its basic form is:

$$
\begin{aligned}
\text { ILI rate }_{t}= & \beta 0+\beta 1 \times \mathrm{RSV}_{t}+\beta 2 \times \operatorname{InfA}(\mathrm{H} 3 \mathrm{~N} 2)_{t}+\beta 3 \\
& \times \operatorname{InfA}(\mathrm{H} 1 \mathrm{~N} 1)_{t}+\beta 4 \times \operatorname{InfB}_{t}
\end{aligned}
$$

where the ILI rate is the population incidence of ILI and the index $t$ represents the running week during the study period. The term $\beta 0$ represents a constant (assumed stable across the time period of study) and $\beta 1-\beta 4$ the regression coefficients for each of the pathogens RSV, influenza A (H3N2), A(H1N1) and influenza B.

The initial model was applied to each country and age strata utilising weekly counts (versus percent of positives, which typically plateaus for many weeks during an epidemic and therefore does not always reflect virus peaks) of positive tests for each of the virus types and subtypes (influenza B, A(H3N2), A(H1N1) and RSV). When one of these terms was not statistically significant ( $p$ value $>0.2$ ) in the model or if the coefficient was negative (suggesting the pathogen prevented ILI and thus not biologically plausible), the term was omitted from the final model.

Model fit and attribution of ILI to each pathogen

The model-predicted and observed weekly ILI rates were compared, and for most age groups and countries, the visual fit of the model, in terms of tracking peaks, was satisfactory (see Fig. 1). Additional regression analyses revealed that the modelled ILI values predicted the observed ILI values reasonably well (average $R^{2}$ value across all countries and age groups was 0.65 ; average standard deviation was 0.07 ). The weekly attribution of ILI rate to each pathogen was generated by multiplying each regression coefficient by its corresponding weekly virus count. We interpret the constant $\beta 0$ to indicate the variability caused by respiratory pathogens other than influenza and RSV combined with measurement error.

Finally, we computed the seasonal attribution by summing the attribution components of each pathogen from week 40 to week 20 of the following year. The percentage of ILI attributed to each virus was calculated using the predicted sum of ILI across the winter weeks as the denominator. The goal of the modelling was to determine the burden of influenza across seasons, age groups and countries; the RSV term in the model controls for the simultaneous contribution of RSV to the ILI signal.

\section{Results}

The results presented in this section are reported as averages across seasons, within each country and age group. Results from individual seasons are reported in Table 3.

England An average of 581 per 100,000 children aged 0-4 visited a physician for ILI each season during 2002-2008. The model attributed an average of $56 \%$ of these consultations (354 per 100,000) to influenza viruses and $16 \%(83$ per 100,000$)$ to RSV. The remaining $28 \%$ of ILI rate was unaccounted for by the model (see Fig. 1 for graphical depiction of the results from this age group). For children aged 5-14 years, 409 per 100,000 consulted a physician for ILI during an average season. The model attributed $77 \%$ of these ILI visits (i.e. 315 per 100,000 ) to influenza viruses and $11 \%(43$ per 100,000$)$ to RSV, while an average $13 \%$ was unattributed by the model.

Italy The Italian study period was, at first, limited to 20042007 to ensure adequate RSV surveillance data. When the RSV terms did not contribute significantly to the model ( $p>0.2$ for both paediatric age groups), the analyses were expanded to include 2003-2008, for which subtyped influenza surveillance data were available. Results of the Italian sensitivity analysis also yielded non-significant RSV terms.

Across these five seasons, an average of 17,748 per 100,000 children aged $0-4(18 \%)$ visited their physician for ILI; the model attributed $52 \%$ of these visits to influenza virus types $\mathrm{A}$ and $\mathrm{B}$ combined, corresponding to $9.8 \%$ of all children aged $0-4$ years. The remaining $48 \%$ of ILI was unattributed by the model (see Fig. 1). Within the 5-14- 
England (0-4 yrs) 2002-2008

๖influenza

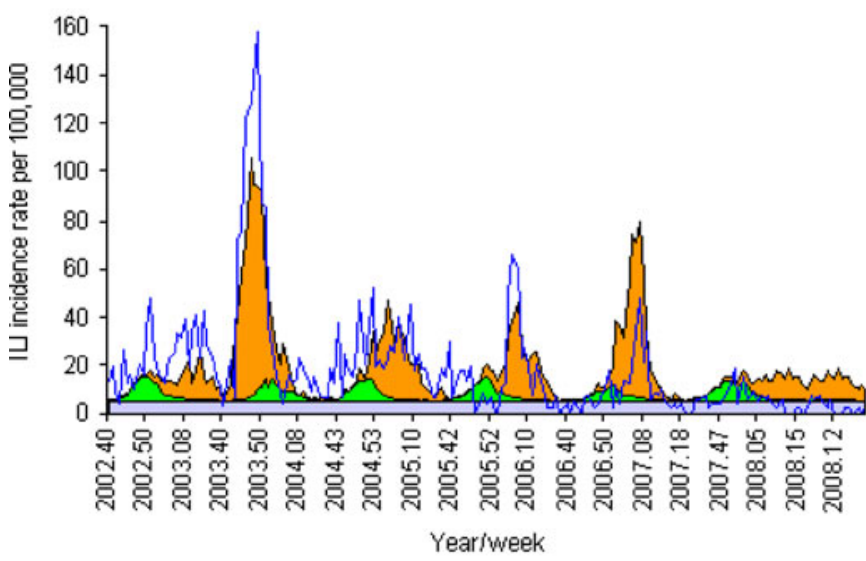

Spain (0-4 yrs) 2002-2008

¿nfluenza A(H3N2) $\square$ Influenza A(H1N1)

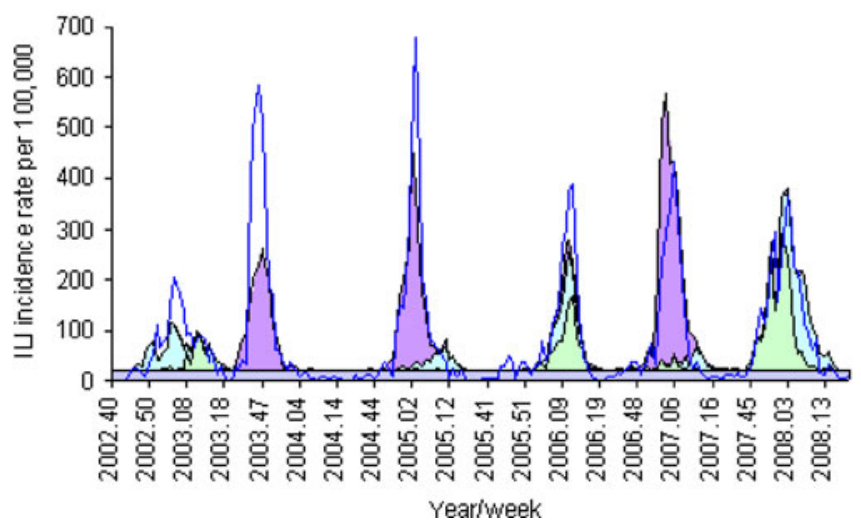

the Netherlands (0-4 yrs) 2002-2008

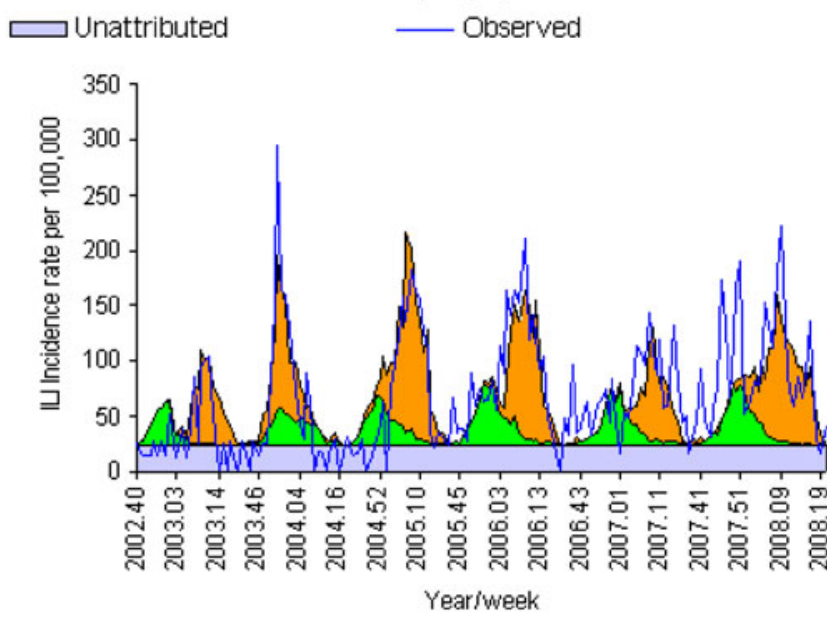

Italy (0-4 yrs) 2003-2008

Influenza B $\square$ Unattributed —observed

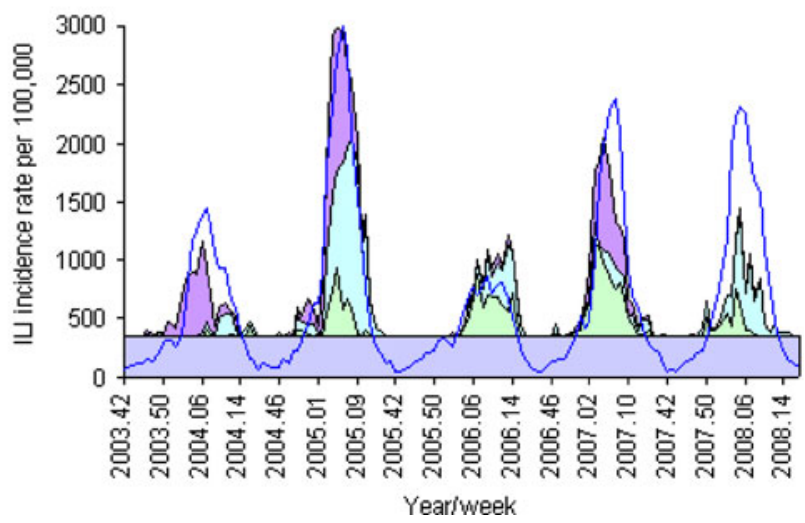

Fig. 1 Observed and predicted physician consultations for ILI by country. In countries where RSV contributed significantly to the observed ILI rate (i.e. England and The Netherlands; upper portion of figure), influenza viruses were grouped together

year-old group, an average of $13 \%$ of children visited their physician for ILI. The model attributed $63 \%$ of these visits (i.e. 8,915 per 100,000 or $8.9 \%$ of the population) to influenza virus types A and B combined, while the remaining ILI variance was unexplained by the model.

The Netherlands During the six seasons 2002-2008, an average of 1,973 per 100,000 children aged $0-4$ years consulted their physician for an ILI. The model attributed $47 \%$ (i.e. 925 per 100,000 ) of ILI to influenza virus and $20 \%$ (i.e. 382 per 100,000 ) to RSV, while $33 \%$ was unattributed (see Fig. 1). A winter average of 970 per 100,000 Dutch children aged 5-14 years visited their physician for ILI. The model attributed $62 \%$ (i.e. 632 per $100,000)$ of the ILI consultations to influenza virus and $13 \%$ (i.e. 121 per 100,000 ) to RSV. An average $25 \%$ ILI variance was unattributed.

Results of the Dutch sensitivity analysis revealed that of the 1,358 per 100,000 ILI visits within the $0-4$-year-old group, the model attributed $70 \%$ (i.e. 964 per 100,000) visits to influenza, $13 \%$ (i.e. 170 per 100,000 ) to RSV and $17 \%$ to other factors. Within the 5-14-year-old group, there was an average 852 ILI visits per 100,000 children. Of these visits, the model attributed $78 \%$ (i.e. 676 per 100,000 ) to influenza. RSV did not contribute significantly to the model $(p=0.4)$, and $22 \%$ of ILI was unattributed.

Spain During 2002-2008, there was a seasonal average of 2,686 per 100,000 children aged $0-4$ years who sought medical attention for ILI. The model attributed $80 \%$ of these cases $(2,156$ per 100,000$)$ to influenza virus types A and B. RSV was not a significant factor in the model ( $p=$ 0.37 ) and $20 \%$ of ILI was unattributed (see Fig. 1). For the 5-14-year-olds, 3,558 per 100,000 children visited their physician with ILI. The model attributed $83 \%$ (i.e. 2,955 per 100,000$)$ of the ILI cases to influenza. RSV was not a significant term in the model ( $p=0.23)$, and $17 \%$ of the ILI variance was unattributed. 
Table 3 Seasonal incidence rates of observed ILI consultations and rates attributed to influenza and RSV by country and age group (per 100,000 children)

\begin{tabular}{|c|c|c|c|c|c|c|c|c|c|c|}
\hline & \multicolumn{2}{|c|}{ Observed ILI } & \multicolumn{2}{|c|}{ Influenza A(H1N1) } & \multicolumn{2}{|c|}{ Influenza A(H3N2) } & \multicolumn{2}{|c|}{ Influenza B } & \multicolumn{2}{|l|}{ RSV } \\
\hline & $0-4$ years & 5-14 years & $0-4$ years & $5-14$ years & $0-4$ years & 5-14 years & $0-4$ years & 5-14 years & $0-4$ years & 5-14 years \\
\hline \multicolumn{11}{|l|}{ England $^{\mathrm{a}}$} \\
\hline $2002-2003$ & 681 & 480 & n.c. & n.c. & 94 & 51 & 67 & 161 & 93 & 48 \\
\hline 2003-2004 & 1,140 & 485 & n.c. & n.c. & 700 & 381 & 7 & 17 & 79 & 41 \\
\hline 2004-2005 & 711 & 414 & n.c. & n.c. & 316 & 172 & 35 & 84 & 81 & 42 \\
\hline 2005-2006 & 490 & 593 & n.c. & n.c. & 141 & 77 & 140 & 337 & 86 & 44 \\
\hline 2006-2007 & 306 & 298 & n.c. & n.c. & 465 & 253 & 7 & 16 & 60 & 31 \\
\hline 2007-2008 & 160 & 186 & n.c. & n.c. & 17 & 9 & 137 & 330 & 97 & 50 \\
\hline \multicolumn{11}{|l|}{ Italy $^{\mathrm{b}}$} \\
\hline 2003-2004 & 14,522 & 10,338 & 251 & 153 & 4,280 & 4,252 & 1,018 & 1,057 & n.s. & n.s. \\
\hline 2004-2005 & 23,242 & 20,608 & 2,303 & 1,400 & 6,943 & 6,898 & 10,302 & 10,702 & n.s. & n.s. \\
\hline $2005-2006$ & 11,347 & 7,927 & 3,513 & 2,135 & 551 & 547 & 2,810 & 2,919 & n.s. & n.s. \\
\hline 2006-2007 & 19,066 & 12,780 & 5,117 & 3,110 & 4,664 & 4,634 & 1,466 & 1,523 & n.s. & n.s. \\
\hline 2007-2008 & 20,562 & 15,546 & 1,554 & 945 & 156 & 155 & 3,991 & 4,145 & n.s. & n.s. \\
\hline \multicolumn{11}{|c|}{ The Netherlands ${ }^{\mathrm{c}}$} \\
\hline $2002-2003$ & 855 & 676 & 28 & n.s. & 286 & 197 & 246 & 190 & 251 & 79 \\
\hline 2003-2004 & 1,510 & 697 & 0 & n.s. & 734 & 505 & 15 & 12 & 332 & 105 \\
\hline 2004-2005 & 1,710 & 1,183 & 83 & n.s. & 641 & 442 & 558 & 431 & 363 & 115 \\
\hline 2005-2006 & 2,472 & 1,374 & 7 & n.s. & 313 & 215 & 714 & 551 & 474 & 150 \\
\hline 2006-2007 & 2,148 & 787 & 50 & n.s. & 547 & 377 & 25 & 19 & 415 & 131 \\
\hline 2007-2008 & 3,142 & 1,104 & 192 & n.s. & 51 & 35 & 1,061 & 819 & 457 & 144 \\
\hline \multicolumn{11}{|l|}{ Spain $^{\mathrm{d}}$} \\
\hline 2002-2003 & 1,842 & 4,075 & 405 & 293 & 37 & 47 & 633 & 1,398 & n.s. & n.s. \\
\hline 2003-2004 & 2,919 & 3,130 & 0 & 0 & 1,544 & 1,950 & 3 & 7 & n.s. & n.s. \\
\hline 2004-2005 & 3,121 & 4,176 & 20 & 15 & 1,968 & 2,486 & 307 & 678 & n.s. & n.s. \\
\hline 2005-2006 & 2,396 & 3,278 & 785 & 568 & 173 & 219 & 595 & 1,314 & n.s. & n.s. \\
\hline 2006-2007 & 2,552 & 2,996 & 112 & 81 & 2,915 & 3,682 & 242 & 534 & n.s. & n.s. \\
\hline 2007-2008 & 3,283 & 3,694 & 1,719 & 1,243 & 52 & 65 & 1,425 & 3,148 & n.s. & n.s. \\
\hline
\end{tabular}

N.B., each winter season was defined as week 40 (October) to week20 (May of the following year)

n.c. negative regression coefficient, n.s. non-significant

${ }^{a}$ Population of English children 0-4 years, 3.0 M; 5-14 years, 6.0 M [29]

${ }^{\mathrm{b}}$ Population of Italian children $0-4$ years, $2.7 \mathrm{M}$; 5-14 years, 5.5 M [11]

${ }^{\mathrm{c}}$ Population of Dutch children 0-4 years, 1.0 M; 5-14 years, $2.0 \mathrm{M}$ [11]

${ }^{\mathrm{d}}$ Population of Spanish children 0-4 years, 2.2 M; 5-14 years, 4.1 M [11]

\section{Discussion}

This paper outlines the design of the EPIA project, the modelling approach, and provides initial modelling results of the burden of medically attended influenza illness in four member countries and for two paediatric age groups. Across the four countries and two age groups investigated in this study, the total ILI burden varied widely between countries, with seasonal averages between $0.4 \%$ and $18 \%$ (mean $=5.2 \%$; median $=2.4 \%$ ) of the populations studied. Influenza epidemics were responsible for most of these ILI events, with seasonal averages of $0.3-9.8 \%$ (mean $=1.6 \%$; median $3.3 \%$ ) observed across countries and age groups. Calculated ILI consultation incidences for the UK and The Netherlands were slightly lower than those reported previously over the period 1986-1997 [14], which is in line with other reports of decreasing incidence of ILI in recent years [9]. With the exception of Spain, data from all countries revealed a higher incidence of influenza-attributed physician consultations in children aged $0-4$ years. These findings are consistent with prior reports of younger children (e.g. infants and toddlers) bearing the highest influenza burden [9].

As seen in Table 3, the results presented from the current study reflect considerable between-country variabil- 
ity in the burden of ILI that is attributed to influenza virus infections, with England having the smallest burden and Italy the highest. These international differences may be influenced by a number of factors. Case definitions, for example, vary between the countries studied (as a function of the fact that many national surveillance networks predate the establishment of the international EISS network). The number of symptoms required to diagnose a patient with ILI as well as specific symptoms themselves are not homogeneous across the countries analysed (i.e. fever is not necessary to diagnose ILI in all countries possibly allowing for a diagnosis in one country that would not otherwise be made in another). Many ILI symptoms are also subjective (e.g. weakness, myalgia, irritability) and diagnosed by self-report or parental reports, which may be sensitive to sociocultural differences between countries (e.g. the outlook of parents on what paediatric symptoms merit a visit to the doctor). Cultural and/or training differences may also play a role on the part of the diagnostician as well as adherence to case definitions. Health systems may also impact health-seeking behaviour through their funding structures, shortages of primary care physicians or other factors that could discourage patients from seeking care such as excessive waiting times, limited physician office hours or appointments.

Provision of primary care in paediatric populations may play a role in health-seeking behaviour as well. Paediatric primary care in Europe can be categorised into three distinct groups: (1) paediatrician-led, (2) GP-led and (3) a combination of the two [22]. Two of the EPIA countries discussed (England and The Netherlands) represent the GPled primary care system, where GPs act as 'gatekeepers' to more specialised care, while Spain has a paediatrician-led system and Italy a combined system (young children are generally cared for by paediatricians while school-age children have the option to see either a paediatrician or a GP [6]). Thus, our reported between-country differences are in agreement with more conservative health-seeking behaviour in GP-led primary care systems and far higher numbers of visits in the paediatrician-led systems. It is also important to note two specific points regarding the situation in Italy, where the highest ILI consultation rates were observed. There are more paediatricians in Italy than any other country in Europe, perhaps creating an environment where consulting a physician is easier for parents in this country compared to others. Additionally, there is a separate, more inclusive, case definition used to diagnose ILI in Italian children. Paediatric-specific ILI case definitions are not employed in the other countries studied.

The finding that an average (across countries and age groups) of $27 \%$ (range 13-48\%) of the observed ILI incidence in children was not attributed to influenza or RSV by the model is in agreement with laboratory-based studies where, depending on the time point during winter, a varying proportion of ILI cases are determined to be due to respiratory pathogens other than influenza and RSV [37]. Thus, pathogens including adenoviruses, metapneumovirus, parainfluenza viruses and Streptococcus pneumoniae may explain some of the residual (unexplained) ILI consultations. However, the importance of influenza driving the ILI signal was evident as the ILI peak always coincided with the peak incidence in influenza virus data. Ideally, it would be useful to expand the model to include additional pathogens.

The low average seasonal attribution to influenza A (H1N1) viruses in England and The Netherlands $(\leq 0.1 \%$ compared to the range of $0.4 \%$ (5-14 years old in Spain) and $2.6 \%(0-4$ years old in Italy)) may reflect its low circulation during the study period 2002-2008 in those countries. Interestingly, our model attributed a relatively small proportion of ILI visits to RSV-a proportion that dropped further in the Dutch data sensitivity analysis when limiting the model period to seasons with discrete (rather than overlapping) RSV and influenza epidemics. Thus, our modelling approach likely tended to overestimate the RSV contribution to outpatient ILI visits.

A direct comparison between previous reports and the current study is not straightforward as the methods employed, range of study years and the outcomes under study are not identical. For example, the study of Fleming et al. [13] was based solely on paediatric RSV and influenza during 1994-2000 and studied a more inclusive outcome, acute respiratory infections. The study of Pitman et al. [30] utilised (among other datasets) national laboratory data for several viruses from 1990 to 2004 and numbers of outpatient consultations for all acute respiratory infections recorded in the General Practice Research Database during the winter of 2002 to 2003. The study of Jansen et al. [20] utilised data from Dutch outpatients using a broader range of diagnoses that would not be captured in the ILI case definition, and utilised GP data from a single regional network rather than a national sentinel network. These three studies $[13,20,30]$ all found larger incidence rates of RSV, which emphasises that there is a need for data encompassing all acute respiratory infections (in order to capture more of the RSV burden), data which is not available in all but one of the EPIA countries (England) and as such could not be presented in this multi-country paper.

One additional limitation of EPIA between-country comparisons is that no standardised protocol was used in the national influenza surveillance systems in Europe; despite EISS collaborations since the 1990s [1], the surveillance systems in each country have a different history, meaning that each country uses a different case definition and different types of reporting physicians (see Table 1), providing multiple opportunities for potential bias in estimated incidence rates. Efforts to harmonise the 
surveillance systems were initiated by the EISS project (e.g. age groups and virological swabbing forms [1]), but differences remain and these almost certainly affect the incidence estimates. Because of this, we have chosen to present results by country and not for all countries grouped together.

With the modelling approach firmly established, the next steps for EPIA will be to extend the analysis to consider more severe outcomes of paediatric influenza, such as hospitalisations and deaths. Also, EPIA plans a number of special subset studies to investigate the consequence of different ILI case definitions in different countries, and the introduction of more viral respiratory pathogens into the model. Furthermore, an expanded age group analysis will be done to study the influenza versus RSV burden in infants. Finally, the emergence of the pandemic A(H1N1) swine-origin influenza virus in the spring of 2009 may inspire countries to use the EPIA model to estimate, near real time, the burden of this emerging virus in the subsequent winters when co-circulation of seasonal influenza viruses and RSV may occur.

The models developed and applied in the EPIA project will facilitate research to derive country-specific results indicating the national burden of paediatric influenza disease in each of the member countries. The overall objective will be to publish this burden of disease data (including pharmacoeconomic analyses) to inform paediatric vaccination recommendations. These analyses concern a very large population in Europe, with more than 52 million inhabitants in the European Union being children aged $0-14$ [8]. Our results so far demonstrate that there was a substantial outpatient febrile respiratory disease burden in some countries of the European Union in an average season between the years of 2002 (2003 in Italy) and 2008: In Italy, 9\% to $10 \%$ of all children aged $0-14$ visited their physician with influenza; in Spain, 2-3\%; and in England and The Netherlands, $<1 \%$.

It is important to bear in mind that our results represent an underestimate of the influenza burden for several reasons: (1) the outcome measured excludes cases of influenza that were treated in other settings (i.e. hospitals and emergency departments) or those that were not professionally attended to, and (2) not all influenza infections are diagnosed as ILI.

As national profiles of paediatric influenza disease burden are determined, new issues and knowledge gaps must be considered. The pharmacoeconomic impact (e.g. cost-benefit analyses) of implementing a new recommendation should be investigated in addition to programmatic issues including feasibility and sustainability (from both financial and logistical perspectives). Finally, although safety, tolerability and efficacy of currently licensed in- fluenza vaccines have been established [21], post-licensure surveillance is crucial to monitor serious adverse events, coverage, effectiveness and the long-term effects on the population $[8,31]$. EPIA aims to provide some of the elements that will help support evidence-based decisions regarding influenza vaccination recommendations for children in Europe.

Acknowledgements We wish to thank Richard Pitman for his assistance developing the model and Marianne van der Sande for her comments to an earlier draft of the manuscript. We would also like to thank all of the physicians and virologists who collected, tested and reported the data used in the analyses and to all EPIA members and their associates for helping make this research possible.

Conflict of interest The EPIA project receives funding from MedImmune. W. John Paget, Catherine Balderston, Laurel Edelman, and Lone Simonsen receive funding from MedImmune via the EPIA project.

EPIA members (February 2010):

Denmark Steffen Glisman, Statens Serum Institut, Copenhagen Anne Mazick, Statens Serum Institut, Copenhagen Kare Molbak, Statens Serum Institut, Copenhagen Lars Nielsen, Statens Serum Institut, Copenhagen

England Alex Elliot, Health Protection Agency, Birmingham Douglas Fleming, Royal College of General Practitioners, Birmingham Rajinder Pnaiser, Royal College of General Practitioners, Birmingham John Watson, Health Protection Agency, London

Finland Timo Vesikari, University of Tampere Medical School, Tampere

Thedi Ziegler, National Public Health Institute, Helsinki

Italy Antonino Bella, Istituto Superiore di Sanita, Rome Isabella Donatelli, Istituto Superiore di Sanita, Rome Fabrizio Pregliasco, Università degli Studi di Milano, Milan Simona Puzelli, Istituto Superiore di Sanita, Rome

The Netherlands Gé Donker, Netherlands Institute for Health Services Research (NIVEL), Utrecht

Adam Meijer, National Institute of Public Health and the Environment (RIVM), Bilthoven

Marianne van der Sande, National Institute of Public Health and the Environment (RIVM), Bilthoven

Scotland Jim McMenamin, Health Protection Scotland, Glasgow Arlene Reynolds, Health Protection Scotland, Glasgow

Spain Pilar Pérez-Breña, Instituto de Salud Carlos III, Madrid Inmaculada Casas, Instituto de Salud Carlos III, Madrid Teresa Lopez Cuadrado, Instituto de Salud Carlos III, Madrid Salvador de Mateo, Instituto de Salud Carlos III, Madrid Amparo Larrauri, Instituto de Salud Carlos III, Madrid Raul Ortiz de Lejarazu Leonardo, Centro Nacional de Gripe, Valladolid 
Tomás Pumarola Suñé, Universitat de Barcelona, Barcelona Tomás Vega Alonso, Health Sentinel Network of Castilla y Leon, Valladolid

Executive Team Catherine Balderston, SDI, USA

Laurel Edelman, SDI, USA

John Paget, NIVEL, The Netherlands

Lone Simonsen, George Washington University, USA

Steering Committee Terho Heikkinen, Turku University, Finland Caterina Rizzo, Istituto Superiore di Sanita, Italy Derek Smith, University of Cambridge, England Catherine Weil-Oliver, Paris Diderot University, UniversiteÏ ParisVII, France

Maria Zambon, Health Protection Agency, UK

Angus Nicoll (ECDC liaison), European Centre for Disease Prevention and Control, Sweden

Open Access This article is distributed under the terms of the Creative Commons Attribution Noncommercial License which permits any noncommercial use, distribution, and reproduction in any medium, provided the original author(s) and source are credited.

\section{References}

1. Arkema JM, Meijer A, Meerhoff TJ, Van Der Velden J, Paget WJ, European Influenza Surveillance Scheme (EISS) (2008) Epidemiological and virological assessment of influenza activity in Europe, during the 2006-2007 winter. Euro Surveill 34:18958

2. Bhat N, Wright JG, Broder KR, Murray EL, Greenberg ME, Glover MJ, Likos AM, Posey DL, Klimov A, Lindstrom SE, Balish A, Medina MJ, Wallis TR, Guarner J, Paddock CD, Shieh WJ, Zaki SR, Sejvar JJ, Shay DK, Harper SA, Cox NJ, Fukuda K, Uyeki TM, Influenza Special Investigations Team (2005) Influenza-associated deaths among children in the United States, 2003-2004. N Engl J Med 24:2559-2567

3. Blanca P, Noëlb G, Dubusc JC, Garnierb JM, Chabrolc B, Minodier P (2005) Caractéristiques d'une épidémie de grippe A chez l'enfant (2003-2004). Impact sur l'activité des services d'urgences et de médecine pédiatriques. Archives de Pediatrie $1: 11-16$

4. Boron ML, Edelman L, Groothuis JR, Malinoski FJ (2008) A novel active respiratory syncytial virus surveillance system in the United States: variability in the local and regional incidence of infection. Pediatr Infect Dis J 12:1095-1098

5. Cohen GM, Nettleman MD (2000) Economic impact of influenza vaccination in preschool children. Pediatrics 5:973-976

6. del Torso S, Bussi R, DeWitt TG (1997) Primary care pediatrics in Italy: eighteen years of clinical care, research, and teaching under a national health service system. Pediatrics 1:E8

7. Dijkstra F, Donker GA, Wilbrink B, Van Gageldonk-Lafeber AB, Van Der Sande MA (2009) Long time trends in influenza-like illness and associated determinants in The Netherlands. Epidemiol Infect 4:473-479

8. Edwards KM (2009) The burden of influenza in children: time for prevention. Clin Infect Dis 49:1022-1024

9. Elliot AJ, Fleming D (2006) Surveillance of influenza-like illness in England and Wales during 1966-2006. Euro Surveill 11(10):249 250

10. European Influenza Surveillance Scheme (2010) European Influenza Surveillance Scheme (EISS). Available online at: http://ecdc. europa.eu/en/Activities/Surveillance/EISN/Pages/home.aspx
11. Fleming DM, Elliot AJ, Cross KW (2007) Morbidity profiles of patients consulting during influenza and respiratory syncytial virus active periods. Epidemiol Infect 7:1099-1108

12. Fleming DM and Miles J (2010) The representativeness of sentinel practice networks. J Public Health 32(1):90-96

13. Fleming DM, Pannell RS, Elliot AJ, Cross KW (2005) Respiratory illness associated with influenza and respiratory syncytial virus infection. Arch Dis Child 7:741-746

14. Fleming DM, Zambon M, Bartelds AI (2000) Population estimates of persons presenting to general practitioners with influenza-like illness, 1987-96: a study of the demography of influenza-like illness in sentinel practice networks in England and Wales, and in The Netherlands. Epidemiol Infect 2:245-253

15. Glezen WP (2009) Benefits of a universal influenza immunization program: more than the reduction in the use of antibiotics. Clin Infect Dis 5:757-758

16. Heijnen MLA, Bartelds AIM, de Jong JC, Rimmelzwaan GF, Wilbrink B (2001) Influenza en RS-virus infecties in winter 2000/ 2001. Infectieziekten Bull 12:50-51

17. Heikkinen T, Silvennoinen H, Peltola V, Ziegler T, Vainionpaa R, Vuorinen T, Kainulainen L, Puhakka T, Jartti T, Toikka P, Lehtinen P, Routi T, Juven T (2004) Burden of influenza in children in the community. J Infect Dis 8:1369-1373

18. Hurwitz ES, Haber M, Chang A, Shope T, Teo S, Ginsberg M, Waecker N, Cox NJ (2000) Effectiveness of influenza vaccination of day care children in reducing influenza-related morbidity among household contacts. JAMA 13:1677-1682

19. Jansen AG, Sanders EA, Hoes AW, van Loon AM, Hak E (2007) Influenza- and respiratory syncytial virus-associated mortality and hospitalisations. Eur Respir J 6:1158-1166

20. Jansen AG, Sanders EA, Wallinga J, Groen EJ, van Loon AM, Hoes AW, Hak E (2008) Rate-difference method proved satisfactory in estimating the influenza burden in primary care visits. J Clin Epidemiol 8:803-812

21. Jefferson T, Rivetti A, Harnden A, Di Pietrantonj C, Demicheli V (2008) Vaccines for preventing influenza in healthy children. Cochrane Database Syst Rev 2:CD004879

22. Katz M, Rubino A, Collier J, Rosen J, Ehrich JH (2002) Demography of pediatric primary care in Europe: delivery of care and training. Pediatrics 5:788-796

23. Larrauri A, de Mateo S, Spanish Influenza Sentinel Surveillance System (2007) Characterisation of swabbing for virological analysis in the Spanish Influenza Sentinel Surveillance System during four influenza seasons in the period 2002-2006. Euro Surveill 5:E5-E6

24. Mangtani P, Cumberland P, Hodgson CR, Roberts JA, Cutts FT, Hall AJ (2004) A cohort study of the effectiveness of influenza vaccine in older people, performed using the United Kingdom general practice research database. J Infect Dis 1:1-10

25. Meerhoff TJ, MacKay WG, Meijer A, Paget WJ, Niesters HG, Kimpen JL, Schellevis F (2008) The impact of laboratory characteristics on molecular detection of respiratory syncytial virus in a European multicentre quality control study. Clin Microbiol Infect 12:1173-1176

26. Meijer A, Valette M, Manuguerra JC, Perez-Brena P, Paget J, Brown C, van der Velden K, Virology Working Group of the European Influenza Surveillance Scheme (2005) Implementation of the community network of reference laboratories for human influenza in Europe. J Clin Virol 2:87-96

27. Mereckiene J, Cotter S, Weber JT, Nicoll A, Levy-Bruhl D, Ferro A, Tridente G, Zanoni G, Berra P, Salmaso S, O'Flanagan D, Gatekeepers Group VENICE (2008) Low coverage of seasonal influenza vaccination in the elderly in many European countries. Euro Surveill 41:19001

28. Neuzil KM, Mellen BG, Wright PF, Mitchel EF Jr, Griffin MR (2000) The effect of influenza on hospitalizations, outpatient 
visits, and courses of antibiotics in children. N Engl J Med 4:225231

29. Office of National Statistics (2008) Office of National Statistics Quarterly population estimate of England and Wales. Office of National Statistics, UK

30. Pitman RJ, Melegaro A, Gelb D, Siddiqui MR, Gay NJ, Edmunds WJ (2007) Assessing the burden of influenza and other respiratory infections in England and Wales. J Infect 6:530-538

31. Poehling KA, Talbot HK, Williams JV, Zhu Y, Lott J, Patterson L, Edwards KM, Griffin MR (2009) Impact of a school-based influenza immunization program on disease burden: comparison of two Tennessee counties. Vaccine 20:2695-2700

32. Reichert TA, Sugaya N, Fedson DS, Glezen WP, Simonsen L, Tashiro M (2001) The Japanese experience with vaccinating schoolchildren against influenza. N Engl J Med 12:889-896

33. Rizzo C, Viboud C, Montomoli E, Simonsen L, Miller MA (2006) Influenza-related mortality in the Italian elderly: no decline associated with increasing vaccination coverage. Vaccine $42-$ 43:6468-6475

34. SAS Institute (1982) SAS user's guide. SAS Institute, Cary

35. StataCorp (2001) Statistical software. StataCorp, College Station
36. The Scientific Panel on Vaccines and Immunisation (2007) Infant and children seasonal immunisation against influenza on a routine basis during inter-pandemic period. The Scientific Panel on Vaccines and Immunisation, Stockholm

37. van Gageldonk-Lafeber AB, Heijnen ML, Bartelds AI, Peters MF, van der Plas SM, Wilbrink B (2005) A case-control study of acute respiratory tract infection in general practice patients in The Netherlands. Clin Infect Dis 4:490-497

38. Velasco L, Soler P (2006) Vigilancia epidemiologica del virus respiratorio sincitial. Casos notificados al Sisterma de Informacion Microbiologica. Temporada 2005-2006. Bol Epidemiol Semanal 14:181

39. Weycker D, Edelsberg J, Halloran ME, Longini IM Jr, Nizam A, Ciuryla V, Oster G (2005) Population-wide benefits of routine vaccination of children against influenza. Vaccine 10:1284-1293

40. World Health Organization (2003) Prevention and control of influenza pandemics and annual epidemics. WHA 10th Plenary Meeting

41. World Health Organization (2009) WHO Vaccine Preventable Diseases Monitoring System-2009 Global Summary. Available online at: http://whqlibdoc.who.int/hq/2009/WHO_IVB_2009_eng_ Part2.pdf 